\section{Controlled growth factor release from synthetic extracellular matrices}

Kuen Yong Lee ${ }^{\star} \ddagger$, Martin C. Peters $\ddagger$, Kenneth W. Anderson $\star \S$ \& David J. Mooney ${ }^{\star} \dagger$

Departments of ${ }^{\star}$ Biologic \& Materials Sciences, $\dagger$ Chemical Engineering, $\ddagger$ Biomedical Engineering, and $\$$ Otolaryngology-Head \& Neck Surgery, University of Michigan, Ann Arbor, Michigan 48109, USA

Polymeric matrices can be used to grow new tissues and organs ${ }^{1,2}$, and the delivery of growth factors from these matrices is one method to regenerate tissues ${ }^{3,4}$. A problem with engineering tissues that exist in a mechanically dynamic environment, such as bone, muscle and blood vessels ${ }^{5,6}$, is that most drug delivery systems have been designed to operate under static conditions. We thought that polymeric matrices, which release growth factors in response to mechanical signals, might provide a new approach to guide tissue formation in mechanically stressed environments. Critical design features for this type of system include the ability to undergo repeated deformation, and a reversible binding of the protein growth factors to polymeric matrices to allow for responses to repeated stimuli. Here we report a model delivery system that can respond to mechanical signalling and upregulate the release of a growth factor to promote blood vessel formation. This approach may find a number of applications, including regeneration and engineering of new tissues and more general drug-delivery applications.

Natural extracellular matrices (ECMs) of tissues are regarded as depots for various growth factors, which are released to cells in the surrounding tissue to affect many physiological processes ${ }^{7}$. For example, release of vascular endothelial growth factor (VEGF) specifically enhances vascularization of tissues ${ }^{8}$. Synthetic and naturally derived polymers can be used similarly as depots and delivery vehicles of protein growth factors ${ }^{9}$. However, most tissues in the body are subjected to mechanical stimuli, and this form of signalling should be considered in the design of polymeric matrices that release growth factors. We thought that growth factor delivery could be designed to respond to compressive stimulation of the matrix, owing to the increased pressure in the carrier resulting from matrix deformation. This mechanically controlled delivery of growth factors might allow the local environment of the delivery device to regulate the local concentration of the factor. This would be particularly advantageous when using growth factor delivery to engineer new vascular tissues or bone. We tested this hypothesis using hydrogel carriers, as hydrogels are frequently used in tissue engineering $^{10}$ and are capable of repeated deformation following compressional loading.

To confirm that compressional loading can regulate drug release from polymeric matrices, we prepared alginate hydrogels containing trypan blue as a model drug molecule and subjected them to repeated compressive loading using a mechanical tester. The release rate and cumulative release of the drug increased during each incident of mechanical loading; however, the cumulative release at the end of each relaxation period was similar to that of control (non-compressed) hydrogels (see Supplementary Information). In this situation, hydrogels do not bind this drug, and the mechanical signalling does not contribute significantly to the release behaviour, owing to rapid depletion of the incorporated drug from the hydrogel.

We subsequently proposed that reversible binding of drugs to the hydrogel carrier, similar to the binding of growth factors to natural ECMs, would allow for a significant effect of cyclic mechanical stimulation on the factor release. VEGF was chosen as a model growth factor because of its use in many clinical indications and its reversible binding interaction with polysaccharides ${ }^{8}$. In the absence of mechanical stimulation, the release rate was fairly constant and the cumulative release of VEGF from the alginate hydrogels increased linearly with time. However, the release rate under mechanical stimulation increased to a value up to five times higher than that of control gels, and this increase could be regulated by the amplitude of the compression (Fig. 1). In this situation, free drug (not bound) would be released after each deformation, but the system would reequilibrate during the subsequent relaxation by the disassociation of bound drug from the hydrogel. The cumulative release profile showed a stepwise increment with mechanical signalling, and the total amount of VEGF released from the hydrogels was up to two times
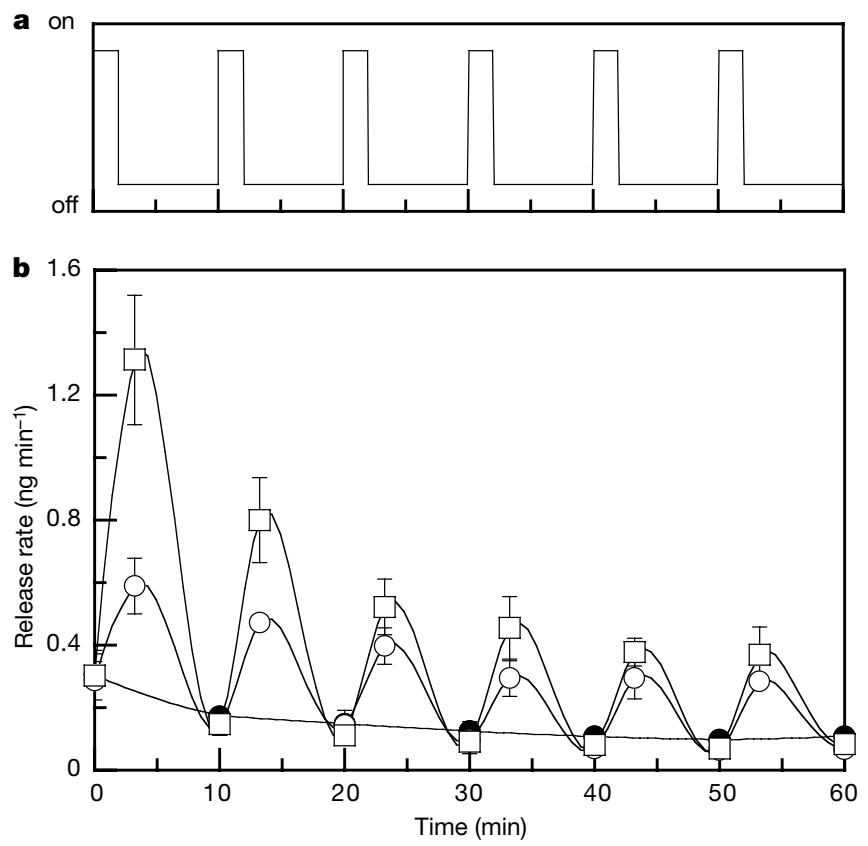

Figure 1 In vitro release profile of VEGF from alginate hydrogels under mechanical stimulation. a, Mechanical stimulation involving six cycles of compression for $2 \mathrm{~min}$, followed by relaxation for $8 \mathrm{~min}$. $\mathbf{b}$, Release rate of VEGF from alginate hydrogels under
$10 \%$ ( $n=4$; open circles) and $25 \%$ ( $n=4$; open squares) strain amplitude, and with no compression ( $n=4$; filled circles) as a control. 
more than that of control conditions, depending on the magnitude of the mechanical input (see Supplementary Information).

Next, we tested the ability of mechanical stimulation to upregulate growth factor release in vivo and affect a local tissue response to show the efficacy and utility of this concept. We used VEGF as a model factor as it is a potent endothelial-cell-specific mitogen; it also enhances neovascularization, which accompanies many physiological and pathological processes ${ }^{11}$, resulting in an increase of granulation tissue thickness and of the number of blood vessels. We subcutaneously implanted hydrogels loaded with VEGF into the dorsal region of severe combined immunodeficient (SCID) mice; we also implanted hydrogels without growth factors as a control. In the absence of growth factors, no remarkable vascularization surrounding the implant was observed with or without mechanical stimulation (Fig. 2a, b). The tissue sections surrounding hydrogels loaded with VEGF showed enhanced vascularization as expected (Fig. 2c, d). Notably, VEGF-loaded hydrogels subjected to cyclic mechanical stimulation showed a statistically significant increase in granulation layer thickness and in vascularization, as compared with non-stimulated VEGF-releasing gels. The thickness of granulation tissue surrounding the implants increased from $33 \pm 8$ to $74 \pm 9 \mu \mathrm{m}$ (mean \pm s.d. $P<0.01$; Fig. $3 \mathrm{a}$ ), and the number of blood vessels in the granulation tissue layer increased from $60 \pm 10$ to 91 \pm 5 under mechanical stimulation ( $P<0.05$; Fig. $3 \mathrm{~b})$.

We investigated this approach further in non-obese diabetic (NOD) mice, as these diabetic animals are frequently used as an animal model for impaired wound healing in humans. We implanted alginate hydrogels containing VEGF into NOD mice after femoral artery ligation to discern whether an increase in collateral circulation could be achieved. Blood vessel formation was quantified after $14 \mathrm{~d}$ of implantation, and enhanced blood vessel formation was observed in mechanically stimulated implant sites (Fig. 4a, b). The density of blood vessels increased from $161 \pm 32$ to $260 \pm 65 \mathrm{~mm}^{-2}$ under cyclic mechanical stimulation $(P<0.05$; Fig. 4c). Hydrogels without VEGF were also used as a control, and no significant blood vessel formation was observed.

Our findings have a number of potential applications for tissue engineering and drug delivery, and also indicate a new general mechanism by which tissues in the body may respond to mechanical signals. It has been proposed that stress-responsive mechanisms include alterations in cellular structure or metabolism ${ }^{12}$, and pre- a

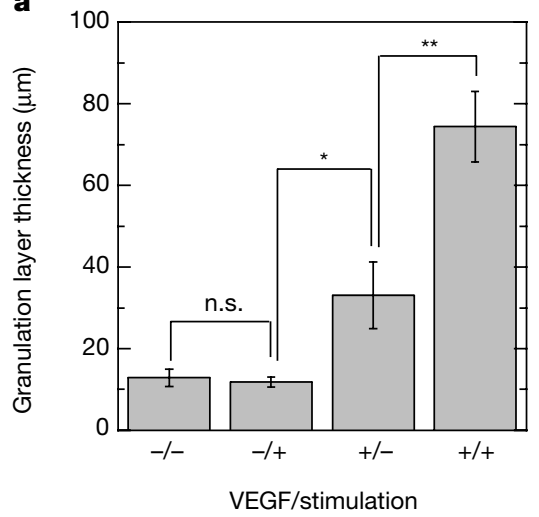

b

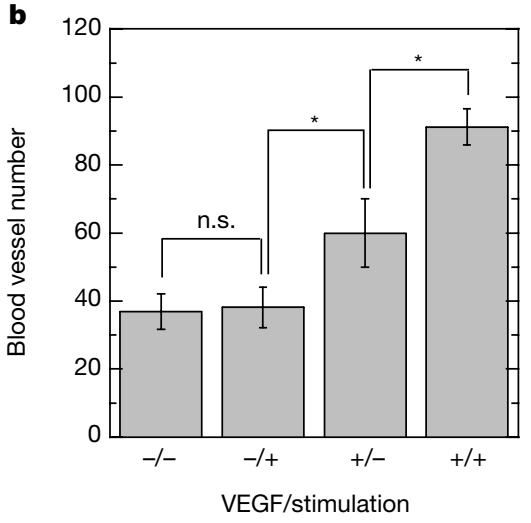

Figure 3 Quantitative analysis of granulation tissue formed in SCID mice. Shown are thickness of granulation tissue (a) and the number of blood vessels (b) at experimental conditions of no VEGF/no mechanical stimulation (-/-), no VEGF/mechanical stimulation $(-/+)$, VEGF incorporated in hydrogel/no mechanical stimulation (+/-) and VEGF incorporated in hydrogel/mechanical stimulation $(+/+)(n=4)$. n.s., no statistical difference. Asterisk indicates statistical significance, $P<0.05$. Double asterisk indicates statistical significance, $P<0.01$.

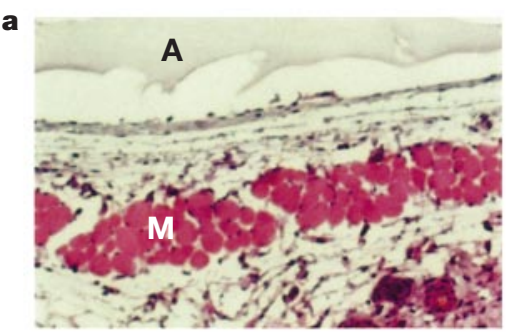

c

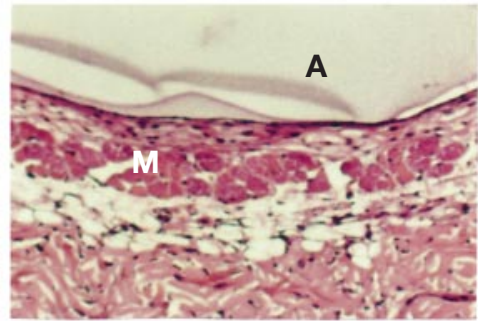

Figure 2 In vivo response to VEGF released from alginate hydrogels under mechanical stimulation. The gels were implanted into the dorsal region of SCID mice for $14 \mathrm{~d}$. Photomicrographs of representative tissue section from each experimental condition: no VEGF/no mechanical stimulation (a); no VEGF/mechanical stimulation (b); VEGF b

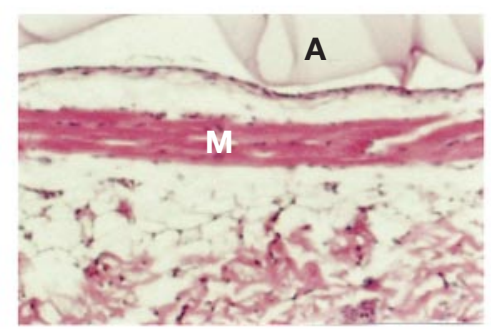

d

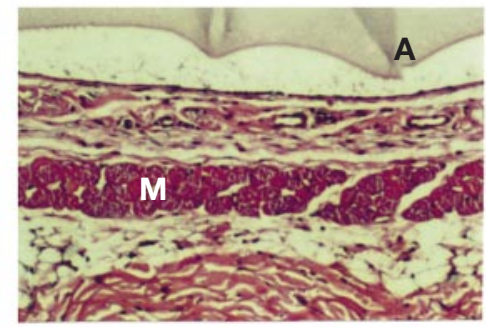

incorporated in hydrogel/no mechanical stimulation (c); and VEGF incorporated in hydrogel/mechanical stimulation (d). Original magnification $\times 100$. A, alginate hydrogel; $\mathrm{M}$, muscle layer. 

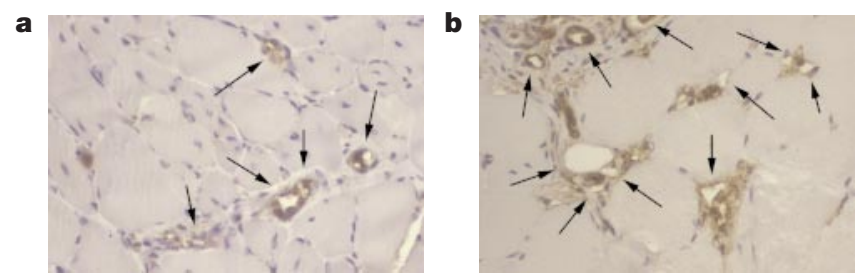

c

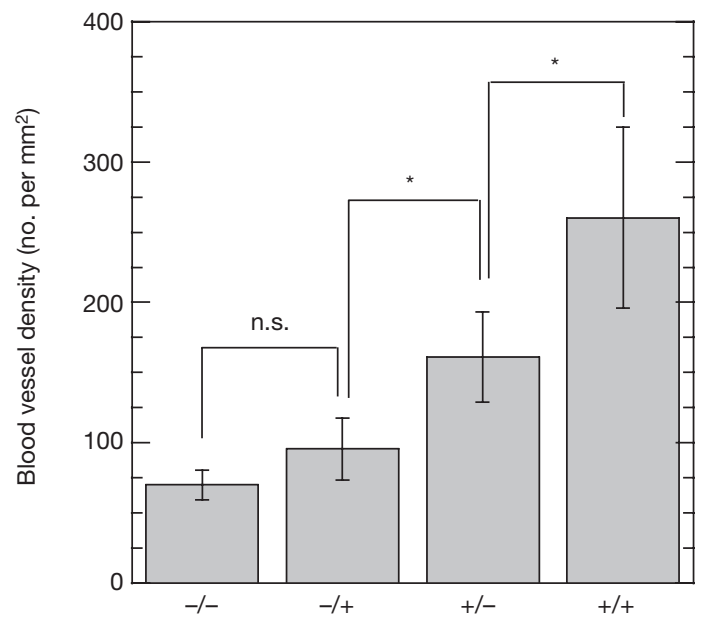

VEGF/stimulation

Figure 4 In vivo response to VEGF-loaded hydrogels implanted into femoral artery ligation site of NOD mice. Shown are photomicrographs of representative tissue section: a, VEGF incorporated in hydrogel/no mechanical stimulation (+/-); b. VEGF incorporated in hydrogel/mechanical stimulation (+/+). c, Blood vessel density (number per $\mathrm{mm}^{2}$ ) at each experimental condition $(n=4)$. Control gels without VEGF under static conditions $(-/-)$ and under mechanical stimulation $(-/+)$ were also implanted. Original magnification $\times 400$. Arrows indicate CD31-stained blood vessels. n.s., no statistical difference. Asterisk indicates statistical significance, $P<0.05$.

vious studies that focused on the role of growth factors have detailed changes in transcription and/or translation of the factors ${ }^{13}$. It is possible, perhaps even likely, that the application of mechanical stress to tissues will lead to an increased release of growth factors through the mechanism that we describe. This may provide an important and previously unrecognized means by which stressed tissues communicate with surrounding tissues, and adapt to their environment. As such, the systems that we have described here may be simply mimicking a critical function of natural ECMs. It may also be possible to develop delivery systems of small drug molecules using this concept. There have been many attempts to develop drugdelivery systems in response to external stimuli such as temperature, $\mathrm{pH}$, ultrasound, and electric or magnetic field ${ }^{14-19}$. However, little effort has been devoted to mechanical stimuli-mediated drug-delivery systems, relative to the significance of these signals in physiological situations. This approach could allow a high level of control over the delivery dose and rate, and might even be incorporated into feedback systems to allow highly precise delivery profiles.

\section{Methods}

\section{In vitro release}

We prepared alginate hydrogels ( $10 \mathrm{~g}$, Pronova) containing $63 \mathrm{mg} \mathrm{CaSO}_{4}$, with either trypan blue ( $4 \mathrm{mg}$, Sigma) or VEGF (10 $\mu \mathrm{g}$, Intergen). We included ${ }^{125}$ I-labelled VEGF (Biomedical Technologies) as a tracer in certain experiments. Gels were cut into disks (12.7 $\mathrm{mm}$ diameter and $2 \mathrm{~mm}$ thick), and pre-swollen in DMEM overnight. The gel disks were put into custom-made sample holders ( $13 \mathrm{~mm}$ diameter and $30 \mathrm{~mm}$ length) with porous stainless-steel supports on the top and bottom of the hydrogels to allow the unimpeded release of the drug from the hydrogel. We compressed the hydrogels with a mechanical tester (MTS, France), and applied 3-6 cycles of compressive loading (10 or $25 \%$ strain; 2 -min strain and 8 -min relaxation). The amount of released trypan blue was spectrophotometrically determined at $588 \mathrm{~nm}$. We measured the release of radiolabelled VEGF by a gamma counter (Packard), and converted it to absolute protein amounts using the known specific activity of ${ }^{125}$ I-labelled VEGF.

\section{Animal models}

Alginate hydrogels $(0.5 \mathrm{~g})$ containing $10 \mu \mathrm{g}$ VEGF and $3.2 \mathrm{mg} \mathrm{CaSO}_{4}$ were cut into disks ( $4.8 \mathrm{~mm}$ diameter and $2 \mathrm{~mm}$ thick) and pre-swollen in DMEM overnight. We anaesthetized animals with an intramuscular injection of $87 \mathrm{mg}$ per $\mathrm{ml}$ ketamine and $2.6 \mathrm{mg}$ per $\mathrm{ml}$ xylazine. The gel disks were subcutaneously implanted into the dorsal region of 7-9-weekold SCID mice in the first model, and a 24 -h recovery period was allowed. Disks were subsequently stimulated daily for $7 \mathrm{~d}$ using $50 \%$ strain amplitude at three cycles of compression for $1 \mathrm{~min}$, followed by relaxation for $1 \mathrm{~min}$ using a custom-made stimulator.

In the second model, the femoral artery in the right hind limb of 7-9-week-old NOD mice was ligated and the exposed arterial ends were tied off with nylon sutures. We then placed a single hydrogel disk directly on the ligation site of each animal and closed the skin with sutures. Twenty-four hours after surgery, we mechanically stimulated the implantation site daily for $7 \mathrm{~d}$, at six cycles of compression for $30 \mathrm{~s}$, followed by relaxation for $90 \mathrm{~s}$. The treatment of experimental animals was in accordance with University of Michigan animal care guidelines, and we observed all NIH animal handling procedures.

After $14 \mathrm{~d}$, the mice in both models were killed and tissues directly surrounding each implant were taken. The tissues were fixed in zinc-formalin solution at $4{ }^{\circ} \mathrm{C}$ overnight, dehydrated through graded ethanol, embedded in paraffin and cut into $4-\mu \mathrm{m}$ sections. To identify blood vessels, the tissue sections from SCID mice were stained with haematoxylin and eosin, and those from NOD mice were immunostained with antibodies raised against mouse CD31 (Pharmingen) using a terminator blocking solution, universal link secondary antibody, streptavadin/horseradish peroxidase (HRP) (Biocare Medical) and 3,3'-diaminobenzidine (Zymed) as described. ${ }^{20}$

We quantified the number of blood vessels and the granulation layer thickness by capturing digital images of tissue sections and analysing them with NIH Image software. The thickness of the newly formed granulation tissue layer was determined between hydrogels and muscle layers. Granulation layer thickness and the number of blood vessels in SCID mice were quantified at $\times 100$ and $\times 400$ magnifications, respectively, and normalized by the interfacial length between the hydrogel and the granulation tissue. We counted the density of blood vessels formed in NOD mice at $\times 400$ magnification. We carried out statistical analysis using Instat software.

Received 11 May; accepted 25 September 2000.

1. Langer, R. \& Vacanti, J. P. Tissue engineering. Science 260, 920-926 (1993).

2. Putnam, A. J. \& Mooney, D. J. Tissue engineering using synthetic extracellular matrices. Nature Med. 2, 824-826 (1996).

3. Shea, L. D., Smiley, E., Bonadio, J. \& Mooney, D. J. DNA delivery from polymer matrices for tissue engineering. Nature Biotechnol. 17, 551-554 (1999).

4. Ripamonti, U. \& Reddi, A. H. Tissue engineering, morphogenesis, and regeneration of the periodontal tissues by bone morphogenetic proteins. Crit. Rev. Oral Biol. Med. 8, 154-163 (1997).

5. Kim, B.-S., Nikolovski, J., Bonadio, J. \& Mooney, D. J. Cyclic mechanical strain regulates the development of engineered smooth muscle tissue. Nature Biotechnol. 17, 979-983 (1999).

6. Niklason, L. E. et al. Functional arteries grown in vitro. Science 284, 489-493 (1999).

7. Vlodavsky, I. et al. Extracellular sequestration and release of fibroblast growth factor: a regulatory mechanism? Trends Biochem. Sci. 16, 268-271 (1991).

8. Neufeld, G., Cohen, T., Gengrinovitch, S. \& Poltorak, Z. Vascular endothelial growth factor (VEGF) and its receptors. FASEB J. 13, 9-22 (1999).

9. Baldwin, S. P. \& Saltzman, W. M. Materials for protein delivery in tissue engineering. Adv. Drug Delivery Rev. 33, 71-86 (1998).

10. Jen, A. C., Wake, M. C. \& Mikos, A. G. Hydrogels for cell immobilization. Biotechnol. Bioeng. 50, 357364 (1996).

11. Plate, K. H., Breiser, G., Weich, H. A. \& Risau, W. Vascular endothelial growth factor is a potential tumor angiogenesis factor in vivo. Nature 359, 845-848 (1992).

12. Chicurel, M. E., Chen, C. S. \& Ingber, D. E. Cellular control lies in the balance of forces. Curr. Opin. Cell Biol. 10, 232-239 (1998).

13. Williams, B. Mechanical influences on vascular smooth muscle cell function. J. Hypertension 16, 1921-1929 (1998).

14. Langer, R. Drug delivery and targeting. Nature 392 (suppl.), 5-10 (1998).

15. Wang, C., Stewart, R. J. \& Kopecek, J. Hybrid hydrogels assembled from synthetic polymers and coiled-coil protein domains. Nature 397, 417-420 (1999).

16. Chen, G. \& Hoffman, A. S. Graft copolymers that exhibit temperature-induced phase transition over a wide range of $\mathrm{pH}$. Nature 373, 49-52 (1995).

17. Mitragorti, S., Blankschtein, D. \& Langer, R. Ultrasound-mediated transdermal protein delivery. Science 269, 850-853 (1995)

18. Kwon, I. C., Bae, Y. H. \& Kim, S. W. Electrically erodible polymer gel for controlled release of drugs. Nature 354, 291-293 (1991).

19. Edelman, E., Brown, L. \& Langer, R. In vitro and in vivo kinetics of regulated drug release from polymer matrices by oscillating magnetic fields. J. Biomed. Mater. Res. 21, 339-353 (1987).

20. Nör, J. E., Christensen, J., Mooney, D. J. \& Polverini, P. J. Vascular endothelial growth factor (VEGF)mediated angiogenesis is associated with enhanced endothelial cell survival and induction of BCL-2 expression. Am. J. Pathol. 152, 375-384 (1999).

Supplementary information is available on Nature's World-Wide Web site

(http://www.nature.com) or as paper copy from the London editorial office of Nature.

\section{Acknowledgements}

We thank the National Institutes of Health for financial support of this research. M.C.P. acknowledges the Whitaker Foundation for a graduate fellowship.

Correspondence and requests for materials should be addressed to D.J.M. (e-mail: mooneyd@umich.edu). 